\title{
Anisotropic flows and shear viscosity of the Quark-Gluon plasma within a transport approach
}

\author{
Salvatore Plumari* \\ Department of Physics and Astronomy, University of Catania, Via S. Sofia 64, I-95125 Catania \\ INFN-Laboratori Nazionali del Sud, Via S. Sofia 62, I-95123 Catania, Italy \\ E-mail: salvatore.plumariect.infn.it
}

Giovanni Luca Guardo, Armando Puglisi, Francesco Scardina, Vincenzo Greco Department of Physics and Astronomy, University of Catania, Via S. Sofia 64, I-95125 Catania INFN-Laboratori Nazionali del Sud, Via S. Sofia 62, I-95123 Catania, Italy

In this talk we discuss the build up of elliptic flow $v_{2}$ and high order harmonics $v_{3}, v_{4}$ and $v_{5}$ for a fluid at fixed $\eta / s$ by mean of an event-by-event transport approach. We discuss the effect of the $\eta / s$ ratio on the build up of the $v_{n}\left(p_{T}\right)$. In particular we study the effect of a temperature dependent $\eta / s$ for two different beam energies: RHIC for Au+Au at $\sqrt{s}=200 \mathrm{GeV}$ and LHC for $\mathrm{Pb}+\mathrm{Pb}$ at $\sqrt{s}=2.76 \mathrm{TeV}$. We find that for the two different beam energies considered the suppression of the $v_{n}\left(p_{T}\right)$ due to the viscosity of the medium have different contributions coming from the cross over or QGP phase. In ultra-central collisions the $v_{n}\left(p_{T}\right)$ show a strong sensitivity to the $\eta / s$ ratio in the QGP phase and this sensitivity increase with the increase of the order of the harmonic.

53rd International Winter Meeting on Nuclear Physics,

26-30 January 2015

Bormio, Italy

\footnotetext{
* Speaker.
} 


\section{Introduction}

The experimental results accumulated in these years in the ultra relativistic heavy ion collisions (uRHICs) first in the experiments conducted at RHIC and more recently at the LHC has shown that the elliptic flow $v_{2}=\left\langle\cos \left(2 \varphi_{p}\right)\right\rangle=\left\langle\left(p_{x}^{2}-p_{y}^{2}\right) /\left(p_{x}^{2}+p_{y}^{2}\right)\right\rangle$, is the largest ever observed in these uRHICs $[1,2]$. The elliptic flow is a measurement of the momentum anisotropy of the emitted particles and it is an observable that encodes information about the transport properties of the matter created in these collisions. Theoretical calculations performed within viscous hydrodynamics framework [3,4] and within transport approach [5, 6, 7] have shown that the large value of $v_{2}$ observed experimentally is consistent with a matter with a very low shear viscosity to entropy density ratio $\eta / s$ and the value is found to be close to the conjectured lower bound for a strongly interacting system, with $\eta / s=1 / 4 \pi$ [8]. While early studies have been focused on elliptic flow generated by the global almond shape of the fireball for non central collisions. In the recent years most of the research activity has been focused on the study of the effects of the fluctuations in the initial geometry due to the fluctuations of the position of the nucleons in the overlap region of the collision. Such fluctuations in the initial geometry are the sources for momentum anisotropies and to a non-zero value of triangular flow and higher order harmonics $v_{n}=\left\langle\cos \left(n \varphi_{p}\right)\right\rangle$. Furthermore, in the recent years the possibility to measure experimentally the event-by-event angular distribution of emitted particle has made possible the measurement of these flow observables at RHIC [9] and at LHC [10]. The comparison between recent event-by-event viscous hydrodynamical calculations and the experimental results for $v_{n}$ seems to confirm a finite but not too large value of $4 \pi \eta / s \sim 1-3$ $[11,12]$. However, small value of $\eta / s \sim 1 / 4 \pi$ is not an evidence of the creation of a QGP phase. A phenomenological estimation of its temperature dependence could give information if the matter created in these collisions undergoes a phase transition [13,14]. Information about a temperature dependence of $\eta / s$ can be achieved studying the $v_{2}\left(p_{T}\right)$ and the high order harmonic $v_{n}\left(p_{T}\right)$ in wider range of energies. Similar studies have been performed using a transport approach but only for the elliptic flow [15]. There are several theoretical indications that $\eta / s$ should have a particular behavior with the temperature[13, 14, 16, 17, 18, 19]. As an example in Fig.1 it is shown a collection of theoretical results about the temperature dependence of $\eta / s$. Fig. 1 shows that in general $\eta / s$ should have a typical behavior of phase transition with a minimum close to the critical temperature $T_{C}[13,14]$. On one hand at low temperature estimations of $\eta / s$ performed in the chiral perturba-

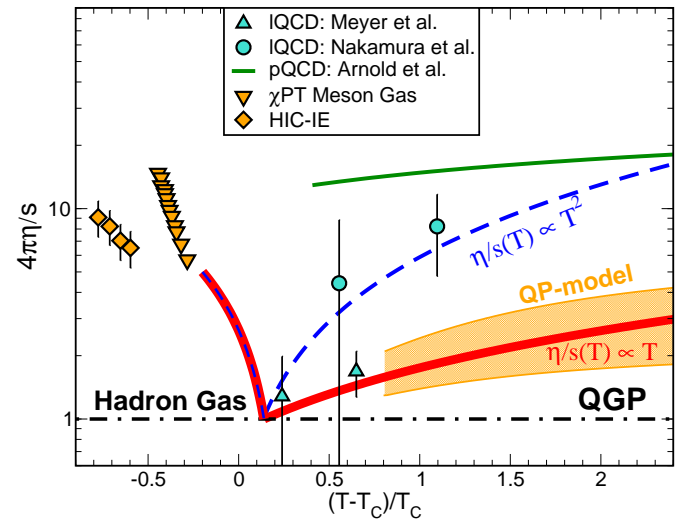

Figure 1: Different parametrizations for $\eta / s$ as a function of the temperature. The orange area refers to the quasi-particle model predictions for $\eta / s$ [20]. The different lines indicate different possible $\mathrm{T}$ dependencies while green line represents the result for the pQCD calculation [21]. Symbols are as in the legend. See the text for more details.

tion theory for a meson gas [16, 17], have shown that in general $\eta / s$ is a decreasing function with 
the temperature, see down-triangles in Fig.1. Similar results for $\eta / s$ have been extrapolated from heavy-ion collisions at intermediate energies, see HIC-IE diamonds in Fig.1. On the other hand at higher temperature $T>T_{c}$ 1QCD calculation have shown but with large error bars that in general $\eta / s$ becomes an increasing function with the temperature [18], see up-triangles and circles in Fig.1. In general due to the large error bars in the lQCD results for $\eta / s$ it is not possible to infer a clear temperature dependence in the QGP phase. The analysis at different energies and centralities of $v_{2}\left(p_{T}\right)$ and the extension to high order harmonics $v_{n}\left(p_{T}\right)$ can give further information about the $\mathrm{T}$ dependence of $\eta / s$. In this proceedings we discuss the build-up of anisotropic flows $v_{n}$ in uRHICs describing the evolution of a system with a fixed $\eta / s(T)$. This is achieved by mean of a transport approach with initial state fluctuations.

\section{Kinetic approach at fixed shear viscosity to entropy density ratio}

In this work to study the dynamical evolution of the initial conditions we employ the transport theory. We perform such simulations using a relativistic transport code developed in these years to perform studies of the dynamics of heavy-ion collisions at both RHIC and LHC energies [5, 7, 22, $23,24,25,26]$. The evolution of the phase-space distribution function $f(x, p, t)$ is given by solving the Relativistic Boltzmann Transport (RBT) equation:

$$
p^{\mu} \partial_{\mu} f(x, p)=\int_{2,1^{\prime}, 2^{\prime}}\left(f_{1^{\prime}} f_{2^{\prime}}-f f_{2}\right)|\mathscr{M}|^{2} \delta^{4}\left(p+p_{2}-p_{1^{\prime}}-p_{2^{\prime}}\right)
$$

In the result shown in this proceeding we have considered only the $2 \leftrightarrow 2$ processes. Where $\int_{2,1^{\prime}, 2^{\prime}}=\int \Pi_{k=2,1^{\prime}, 2^{\prime}} d^{3} p_{k} / 2 E_{k}(2 \pi)^{3}$ and $\mathscr{M}$ denotes the transition amplitude for the elastic processes and it is directly linked to the differential cross section $|\mathscr{M}|^{2}=16 \pi s\left(s-4 M^{2}\right) d \sigma / d t$ with $s$ the Mandelstam invariant. Numerically we solve the RBT equation using the so called test particle method and the collision integral is solved by using Monte Carlo methods based on the stochastic interpretation of transition amplitude [27, 5, 23]. In the standard use of the transport theory one fixes the microscopical details like cross sections of the processes and one can study the effect of the microscopical details on the observables. In the following discussion we invert the description not in terms of fixed cross section $\sigma_{t o t}$ but in terms of fixed $\eta / s$. In this way we get an approach which provides a tool that permit to study the effect of $\eta / s$ on the observables like the $v_{n}\left(p_{T}\right)$ in a wider range of $\eta / s$ and $p_{T}$ than hydrodynamical framework. A more detailed discussion about this approach can be found in previous papers, see [5, 24, 25].

In order to study the dynamical evolution of the fireball with a certain $\eta / s(T)$ we determine locally in space and time the total cross section $\sigma_{t o t}$ needed to have the wanted local viscosity. In this approach it is necessary to know the correct expression for the shear viscosity $\eta$ in terms of microscopical details. In the Chapmann-Enskog theory and for a pQCD inspired cross section, typically used in parton cascade approaches $[28,29,5,30,31,27,6]$, with $d \sigma / d t \sim \alpha_{s}^{2} /\left(t-m_{D}^{2}\right)^{2}$, the $\eta / s$ is given by the following expression:

$$
\eta / s=\frac{1}{15}\langle p\rangle \tau_{\eta}=\frac{1}{15} \frac{\langle p\rangle}{g(a) \sigma_{t o t} \rho},
$$

where $a=m_{D} / 2 T$, with $m_{D}$ being the screening mass regulating the angular dependence of the cross section, while $g(a)$ is the proper function accounting for the pertinent relaxation time $\tau_{\eta}^{-1}=$ 


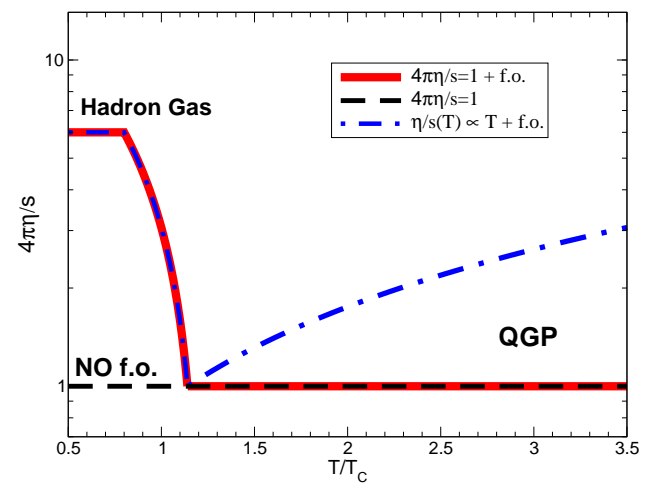

Figure 2: Different parametrization of $\eta / s(T)$. Black dashed line refer to $4 \pi \eta / s=1$ during all the evolution. Red solid line refers to the inclusion of kinetic f.o. for $T \leq 1.2 T_{C}$. Finally, blue dot dashed line refer to the inclusion of linear temperature dependence for $T>1.2 T_{C}$ and the kinetic f.o. at lower temperature.

$g(a) \sigma_{t o t} \rho$ associated to the shear transport coefficient and it is given by:

$$
g(a)=\frac{1}{50} \int d y y^{6}\left[\left(y^{2}+\frac{1}{3}\right) K_{3}(2 y)-y K_{2}(2 y)\right] h\left(\frac{a^{2}}{y^{2}}\right)
$$

where $K_{n}$-s are the Bessel functions and the function $h$ relate the transport cross section to the total cross section $\sigma_{t r}(s)=\sigma_{t o t} h\left(m_{D}^{2} / s\right)$ with $h(\zeta)=4 \zeta(1+\zeta)[(2 \zeta+1) \ln (1+1 / \zeta)-2]$. Therefore the total cross section is evaluated locally by mean the Eq.2.2. In order to study the role of the $\eta / s$ ratio and its temperature dependence we have performed three different calculations: one with a constant $4 \pi \eta / s=1$ during all the evolution of the system dashed line in Fig.2 another one with $4 \pi \eta / s=1$ at higher temperature in the QGP phase and an increasing $\eta / s$ in the cross over region towards the estimated value for hadronic matter $4 \pi \eta / s \approx 6$ shown by solid line in Fig.2. In the following discussion the term f.o. take into account the increase of $\eta / s$ at low temperature. The third one is shown in Fig.2 by the dot dashed line. In this case we consider the increase of $\eta / s$ at higher temperature with a linear temperature dependence and a minimum close to the critical temperature with a temperature dependence similar to that is expected from general considerations as shown in Fig.1.

\section{Initial conditions}

The main novelty in the present paper is the implementation of initial state fluctuations in a transport cascade approach. In this work we will show the results for the two systems $A u+A u$ collisions at $\sqrt{s_{N N}}=200 \mathrm{GeV}$ produced at RHIC and $\mathrm{Pb}+\mathrm{Pb}$ collisions at $\sqrt{s_{N N}}=2.76 \mathrm{TeV}$ at LHC. In order to generate an event by event initial profile we use the Monte-Carlo Glauber model. In this model the Woods-Saxon distribution is used to sample randomly the positions of the nucleons in the two colliding nucleus $A$ and $B$. In this way a discrete distribution for these nucleons is generated. Within this method two nucleons collide each other if the relative distance in the transverse plane is $d_{T} \leq \sqrt{\sigma_{N N} / \pi}$ where $\sigma_{N N}$ is the nucleon-nucleon cross section. In this way we can determine the number of collision $N_{\text {coll }}$ and participant nucleons $N_{\text {part }}$. In our calculation 

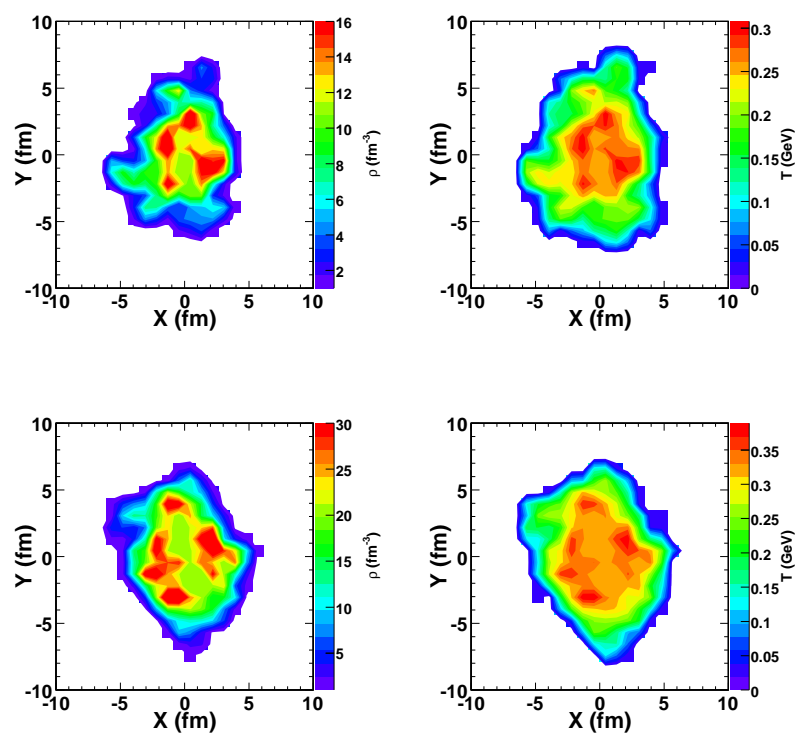

Figure 3: In the left column it is shown the initial transverse density $\rho_{T}(x, y)$ at mid rapidity for $A u+A u$ at $\sqrt{s_{N N}}=200 \mathrm{GeV}$ (upper panel) and $\mathrm{Pb}+\mathrm{Pb}$ at $\sqrt{s_{N N}}=2.76 \mathrm{TeV}$ (lower panel). In the right column it is shown the corresponding initial temperature in transverse plane. These plots are for $b=7.5 \mathrm{fm}$.

we have used $\sigma_{N N}=4.2 \mathrm{fm}^{2}$ for RHIC and $\sigma_{N N}=7.0 \mathrm{fm}^{2}$ for LHC. The discrete distribution of nucleons is converted into a smooth one by assuming for each nucleon a gaussian distribution centered in the nucleon position. In our model we choose to convert the information of the nucleon distribution into the density in the transverse plane $\rho_{T}(x, y)$ which is given by the following sum

$$
\rho_{T}(x, y)=C \sum_{i=1}^{N_{\text {part }}} \exp \left[-\frac{\left(x-x_{i}\right)^{2}+\left(y-y_{i}\right)^{2}}{2 \sigma_{x y}^{2}}\right]
$$

where $C$ is an overall normalization factor fixed by the longitudinal distribution $d N / d y$ while $\sigma_{x y}$ is the Gaussian width and in the following calculations it has been fixed to $\sigma_{x y}=0.5 \mathrm{fm}$. In our calculation we have assumed a longitudinal boost invariant distribution from $y=-2.5$ to $y=2.5$. In the first column of Fig.3 it is shown the contour plot of the initial transverse density at mid rapidity for a given event with impact parameter $b=7.5 \mathrm{fm}$. The upper panel refers to the system $A u+A u$ at $\sqrt{s_{N N}}=200 \mathrm{GeV}$ and the lower panel to $P b+P b$ at $\sqrt{s_{N N}}=2.76 \mathrm{TeV}$. In this way we generate an initial transverse profile that change event by event. Once the transverse density $\rho_{T}(x, y)$ has been fixed the initial anisotropy in coordinate space can be evaluated and it is quantified in terms of the following coefficients $\varepsilon_{n}=\sqrt{\left\langle r_{T}^{n} \cos (n \phi)\right\rangle^{2}+\left\langle r_{T}^{n} \sin (n \phi)\right\rangle^{2}} /\left\langle r_{T}^{n}\right\rangle$ where $r_{T}=\sqrt{x^{2}+y^{2}}$ and $\phi=\arctan (y / x)$ are the polar coordinates in the transverse plane. In Fig. 4 we have shown the $\varepsilon_{2}, \varepsilon_{3}$ and $\varepsilon_{4}$ as a function of the number of participant $N_{\text {part }}$.

For the initialization in momentum space at RHIC (LHC) energies we have considered for partons with transverse momentum $p_{T} \leq p_{0}=2 \mathrm{GeV}(3 \mathrm{GeV})$ a thermalized spectrum in the transverse plane. Assuming the local equilibrium the initial local temperature in the transverse plane $T(x, y)$ is evaluated by using the standard thermodynamical relation $\rho_{T}(x, y)=\gamma T^{3} / \pi^{2}$ with $\gamma=35$. In the 

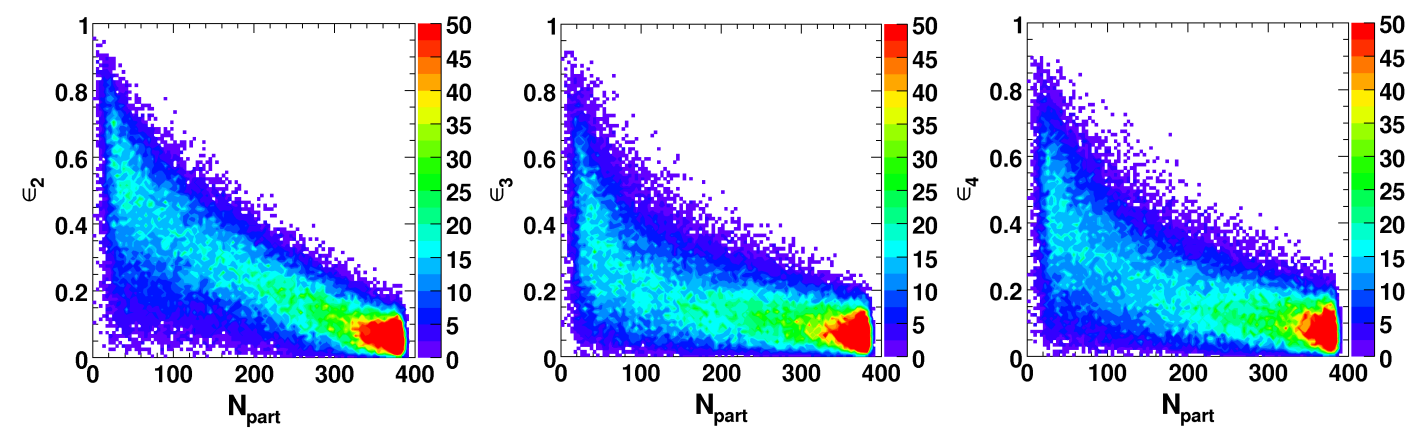

Figure 4: In the left, middle and right panel it is shown the coefficient $\varepsilon_{n}$ as a function of the participant nucleons $N_{\text {part }}$ respectively for $n=2,3$ and 4 .

right column of Fig. 3 it is shown the corresponding initial local temperature in transverse plane. As shown in the central region of the fireball for mid peripheral collision we can reach temperature $T \approx 300 \mathrm{MeV}$ at RHIC and $T \approx 400 \mathrm{MeV}$ at LHC. While for partons with $p_{T}>p_{0}$ we have assumed the spectrum of non-quenched minijets according to standard NLO-pQCD calculations with a power law shape. The initial transverse momentum of the particles is distributed uniformly in the azimuthal angle. We fix the initial time of the simulation to $\tau_{0}=0.6 \mathrm{fm} / \mathrm{c}$ for RHIC and $\tau_{0}=0.3 \mathrm{fm} / \mathrm{c}$ for LHC. In the following discussion, we will consider two different types of initial conditions. One of them consist in a fixed initial distribution by using the standard Glauber model as used in previous works, see $[5,7,22,15]$. The second one consist of an initial profile changing event by event according to the MC glauber model as discussed before.

\section{Effects of $\eta / s$ on the $v_{n}\left(p_{T}\right)$}

In our simulations we have used $N_{\text {event }}=500$ events for each centrality class. This number is enough to get solid results for the spectra, differential elliptic flow and high order flow coefficients $v_{n}\left(p_{t}\right)$. For the study of the correlations between the initial $\varepsilon_{n}$ and the final $v_{n}$ that will be shown in the next section we have extended this analysis to 1000 events. The inclusion of the initial state fluctuations introduce a further difficulties because in order to get stable results we need to have a good sampling of the initial geometry event by event and a good grid resolution. We found the convergency for a grid with $A_{T}=0.12 \mathrm{fm}^{2}$ of transverse area and $N_{\text {test }}=2 \cdot 10^{6}$ total number of test particles. The elliptic flow $v_{2}\left(p_{T}\right)$ and the high order harmonics $v_{3}\left(p_{T}\right)$ and $v_{4}\left(p_{T}\right)$ have been calculated using the formula: $v_{n}=\left\langle\cos \left[n\left(\phi-\Psi_{n}\right)\right]\right\rangle$ where the event-plane angles $\Psi_{n}$ are given by $\Psi_{n}=\frac{1}{n} \arctan (\langle\sin (n \phi)\rangle /\langle\cos (n \phi)\rangle)$ In this section first we discuss the effect of the initial state fluctuations on the final $v_{2}\left(p_{T}\right)$.In Fig.5 we compare the differential elliptic flow $v_{2}\left(p_{T}\right)$ obtained with an initial state that change event by event according to the MC Glauber model (solid lines) as discussed in details in the previous section with the one obtained for the case with an averaged initial profile (dashed lines). These results are for $A u+A u$ collisions at $\sqrt{s}=200 \mathrm{MeV}$ and for two different impact parameters $b=2.5 \mathrm{fm}$ and $b=7.5 \mathrm{fm}$. In these calculations we have considered $4 \pi \eta / s=1$ at high temperature and an increasing $\eta / s$ at lower temperature as shown by the red solid line in Fig.2. As shown for mid peripheral collision (with $b=7.5 \mathrm{fm}$ ) the effect of the 


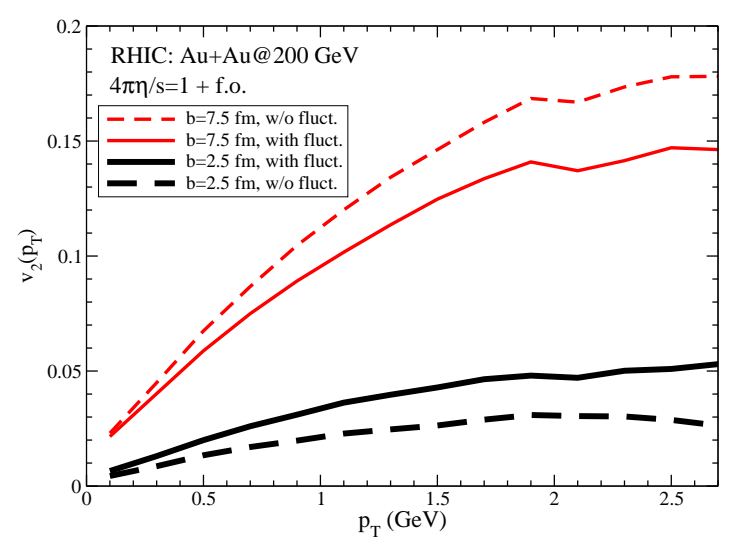

Figure 5: Results for $A u+A u$ collisions at $\sqrt{s_{N N}}=200 \mathrm{GeV}$ for mid rapidity. Differential elliptic flow $v_{2}\left(p_{T}\right)$ at mid rapidity. The solid lines refer to the case with initial state fluctuations the dashed lines are for the case without initial fluctuations. Thick lines are for $b=2.5 \mathrm{fm}$ while thin lines for $b=7.5 \mathrm{fm}$.

fluctuations in the initial geometry is to reduce the $v_{2}\left(p_{T}\right)$ of about $15 \%$. The reduction is related to the fact that for an irregular geometry in the transverse plane the pressure gradients generate a small counter-flow towards the inner part of the fireball reducing the azimuthal anisotropy in momentum space due to the global almond shape. On contrary, We observe an opposite effect for the elliptic flow in more central collisions. In fact as shown by the black thick solid and dashed lines in the left panel of Fig.4 the effect of the fluctuations in the initial geometry is to produce a larger $v_{2}\left(p_{T}\right)$. These results are qualitatively in agreement to that has been obtained also in viscous hydrodynamics calculations [32]. Furthermore the introduction of the fluctuations in the initial geometry play the role to generate the higher order harmonics in particular the odd harmonics which were absent by symmetry in the averaged initial configuration. Differential flow coefficients $v_{n}\left(p_{T}\right)$ are observables that carry out information about the fireball created in the heavy ion collisions in particular they are sensitive to the transport properties of the medium like the $\eta / s$ ratio. In the following discussion we will study the effect of the $\eta / s$ on the build up of the elliptic flow $v_{2}\left(p_{T}\right)$ and on the high order harmonics $v_{3}\left(p_{T}\right)$ and $v_{4}\left(p_{T}\right)$. With $v_{n}\left(p_{T}\right)$ we mean the root mean square $\sqrt{\left\langle v_{n}^{2}\right\rangle}$ as it has been done in experimental data using the event plane method. In the left panel of Fig.6 it is shown the elliptic flow $v_{2}\left(p_{T}\right)$ (green thick lines) and the $v_{4}\left(p_{T}\right)$ (blue thin lines) at mid rapidity and for $(20-30) \%$ centrality for both RHIC $A u+A u$ at $\sqrt{s}=200 \mathrm{GeV}$ (left panel) and LHC $P b+P b$ at $\sqrt{s}=2.76 T e V$ (right panel). In general in agreement with what has been obtained in viscous hydrodynamical calculations, the increase of the viscosity of the medium has the effect to reduce both $v_{2}$ and $v_{4}$. As shown at RHIC energies the $v_{2}\left(p_{T}\right)$ is sensitive to the increase of the $\eta / s$ at lower temperature close to the cross over region. In particular the effect is a reduction of the elliptic flow of about $17 \%$. A similar behavior we observe for the 4-th harmonic $v_{4}\left(p_{T}\right)$ where again we have a reduction due to the increase of $\eta / s$ at lower temperature but the effect in this case is larger then the previous one of about $30 \%$. The different sensitivity of $v_{2}\left(p_{T}\right)$ and $v_{4}\left(p_{T}\right)$ to the $\eta / s$ can be attributed to their different formation time, $t_{v_{4}}>t_{v_{2}}$ [30]. In fact each harmonics $v_{n}$ start to develop at different times. In particular $v_{4}$ start to develop later then $v_{2}$. This means that different harmonics probe mainly different temperatures and different value of the $\eta / s$ ratio. 

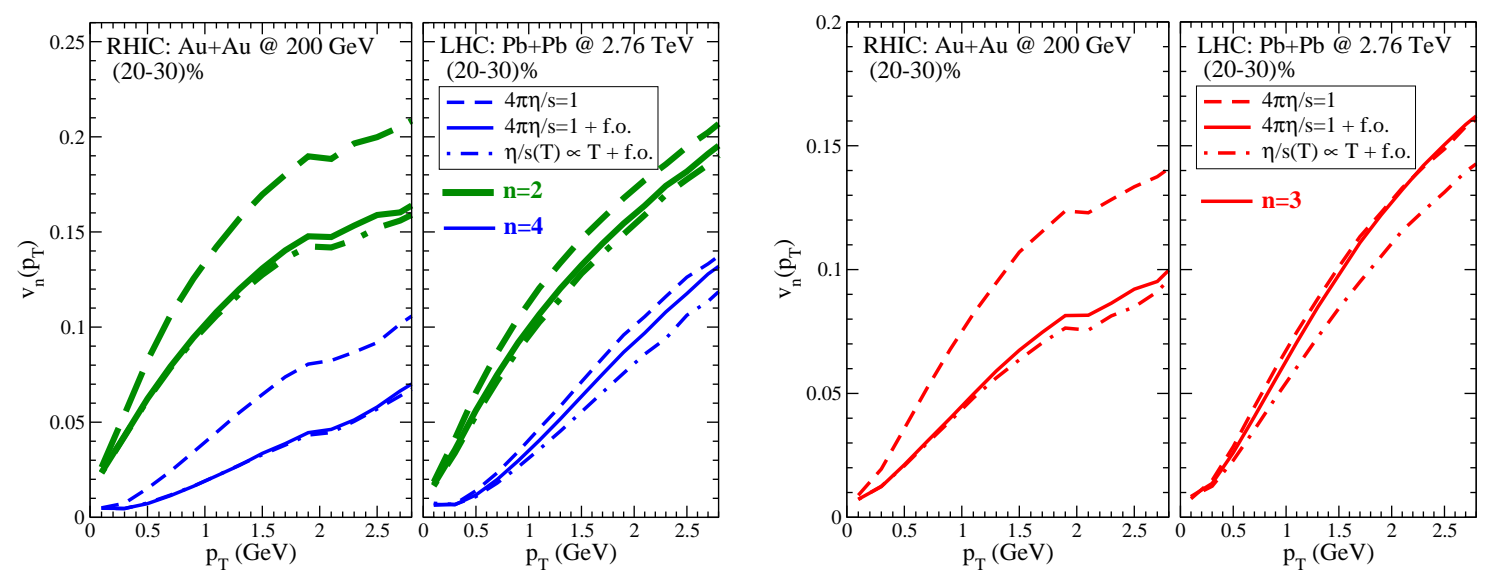

Figure 6: Left panel: differential $v_{2}\left(p_{T}\right)$ (thick green lines) and $v_{4}\left(p_{T}\right)$ (thin blue lines) at mid rapidity and for $(20-30) \%$ collision centrality. The comparison is between the two systems: $A u+A u$ at $\sqrt{s}=200 \mathrm{GeV}$ (left) and $P b+P b$ at $\sqrt{s}=2.76 \mathrm{TeV}$ (right). The dashed lines refer to the case with a constant $\eta / s=(4 \pi)^{-1}$ during all the evolution. The solid lines refer to the case with $\eta / s=(4 \pi)^{-1}$ at higher temperature and with an increasing $\eta / s$ ratio at lower temperature while the dot dashed lines to the case with $\eta / s \propto T$ at higher temperature and with an increasing $\eta / s$ ratio at lower temperature. Right panel: differential $v_{3}\left(p_{T}\right)$ in red lines with the same legend as in the left panel.

On the other hand at LHC energies, the $v_{2}\left(p_{T}\right)$ is almost unaffected by the increase of $\eta / s$ ratio at low temperature as we can see comparing the thick dashed line with the solid one. Moreover we observe that the increase of $\eta / s$ at lower temperature has a more sensitive effect on the $v_{4}\left(p_{T}\right)$ with a reduction of about $10 \%$, see blue solid and dashed lines. Again the different sensitivity to the $\eta / s$ ratio at lower temperature at $\mathrm{LHC}$ are consistent with the results obtained at RHIC and related to the different formation time of the harmonics.

On the other hand the greater sensitivity observed at RHIC to the $\eta / s$ value at low temperature compared to LHC is related to the different life time of the fireball. In fact the life time of the fireball at LHC is greater than that at RHIC, 8-10 $\mathrm{fm} / \mathrm{c}$ at LHC against 4-5 $\mathrm{fm} / \mathrm{c}$ at RHIC. In general this means that at RHIC energies the $v_{n}$ have not enough time to fully develop in the QGP phase. On contrary at LHC we have that the $v_{n}$ develops almost completely in the QGP phase and therefore they are less sensitive to the dynamics at lower $\mathrm{T}$. Therefore it is interesting to study the effect of an $\eta / s(T)$ in the QGP phase. In Fig.6 by the dot-dashed lines it is shown such effect. As we can see comparing the solid lines with the dot dashed ones the effect of an increasing $\eta / s$ at higher temperature has the effect to reduce the $v_{2}\left(p_{T}\right)$ and $v_{4}\left(p_{T}\right)$ produced and the sensitivity increase with the increase of the collision energy. In fact the $v_{4}\left(p_{T}\right)$ at LHC is sensitive to the change of $\eta / s$ at higher temperature while at RHIC energies the $v_{4}$ is unaffected by this change. In the lower panel of Fig.6 it is shown the triangular flow $v_{3}\left(p_{T}\right)$ (red lines) at mid rapidity for $(20-30) \%$ centrality and for both RHIC $A u+A u$ at $\sqrt{s}=200 \mathrm{GeV}$ (left panel) and LHC $P b+P b$ at $\sqrt{s}=2.76 \mathrm{TeV}$ (right panel). In agreement with what has been obtained for the even harmonics $v_{2}\left(p_{T}\right)$ and $v_{4}\left(p_{T}\right)$, we observe at RHIC energies a reduction of $v_{3}\left(p_{T}\right)$ due to the increase of the $\eta / s$ at low temperature with a reduction of about $25 \%$, while at LHC it is almost insensitive to the change of $\eta / s$ in the cross over region. However we observe that at LHC the third and fourth harmonics are more sensitive to the change of $\eta / s(T)$ respect the elliptic flow with a deviation of 


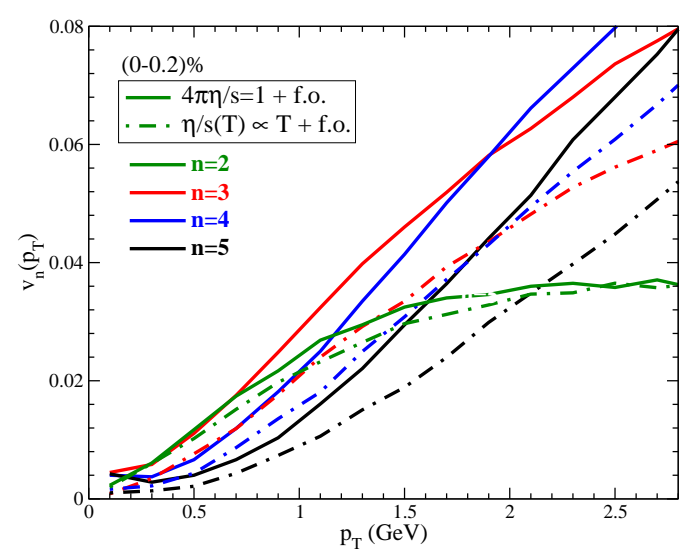

Figure 7: $v_{n}\left(p_{T}\right)$ at mid rapidity for $(0-0.2) \%$ centrality and for $P b+P b$ collisions at $\sqrt{s_{N N}}=2.76 \mathrm{TeV}$. Different colors refer to different harmonics while solid lines correspond to $4 \pi \eta / s=1$ in QGP phase and f.o. and dot dashed lines to $\eta / s \propto T$ in the QGP phase and f.o..

about $10 \%$ and $15 \%$ for $v_{3}$ and $v_{4}$ against $5 \%$ for $v_{2}$.

In Fig.7 it is shown the $v_{n}\left(p_{T}\right)$ for $n=2,3,4$ and $n=5$ at LHC energies and for ultra central collisions. As we can see at low $p_{T}$ the $v_{n}\left(p_{T}\right)$ are much flatter for larger $n$ in agreement with that has been obtained in hydrodynamic calculations where $v_{n}\left(p_{T}\right) \propto p_{T}^{n}$. At hight $p_{T}$ we observe that the $v_{2}\left(p_{T}\right)$ shows a saturation while for $n \geq 3 v_{n}\left(p_{T}\right)$ increase linearly with $p_{T}$. In particular the sensitivity to the value of $\eta / s$ in the QGP phase increase with the increasing the order of the harmonics $n$ in agreement with the fact that viscous corrections to $v_{n}\left(p_{T}\right)$ increase with the harmonics [26]. We observe that reduction of $v_{n}\left(p_{T}\right)$ due to the increase of $\eta / s$ in the QGP phase (dot dashed lines) is enhanced for central collisions with a reduction of about $25 \%-30 \%$ against a reduction of about $10 \%$ for mid peripheral collisions. In particular in central collisions higher harmonics acquire a larger sensitivity to the value of the viscosity in the QGP phase.

\section{Conclusions}

Using an event-by-event transport approach we have investigated the build up of the anisotropic flows $v_{n}\left(p_{T}\right)$ for $n=2,3,4$. We have studied the effect of $\eta / s$ ratio on $v_{n}\left(p_{T}\right)$ for two different beam energies: at RHIC for $A u+A u$ collisions at $\sqrt{s}=200 \mathrm{GeV}$ and at $\mathrm{LHC}$ for $\mathrm{Pb}+\mathrm{Pb}$ collisions at $\sqrt{s}=2.76 \mathrm{TeV}$. We have shown that at RHIC the $v_{n}\left(p_{T}\right)$ are more sensitive to the value of $\eta / s$ at low temperature and the sensitivity increase with the order of the harmonics. At LHC we get a different effect, the $v_{n}\left(p_{T}\right)$ are more sensitive to the increase of $\eta / s$ in the QGP phase at high temperature. We found an enhancement of the sensitivity of the $v_{n}\left(p_{T}\right)$ respect to the value of $\eta / s$ in the QGP phase in ultra central collisions and this sensitivity increase with $n$.

\section{Acknowledgments}

V.Greco, S. Plumari, G.L. Guardo and F. Scardina acknowledge the support of the ERC-StG Grant under the QGPDyn project. 


\section{References}

[1] J. Adams et al. (STAR Collaboration), Nucl.Phys. A757 (2005) 102-183.

[2] K. Aamodt K et al. (ALICE Collaboration), Phys.Rev.Lett. 105 (2010) 252302.

[3] P. Romatschke and U. Romatschke, Phys.Rev.Lett. 99 (2007) 172301.

[4] H. Song and U. W. Heinz, Phys.Rev. C78 (2008) 024902.

[5] G. Ferini, M. Colonna, M. Di Toro and V. Greco, Phys.Lett. B670 (2009) 325-329.

[6] Z. Xu and C. Greiner, Phys.Rev. C79 (2009) 014904.

[7] S. Plumari and V. Greco, AIP Conf.Proc. 1422 (2012) 56-61.

[8] P. Kovtun,D. Son and A. Starinets, Phys.Rev.Lett. 94 (2005) 111601.

[9] A. Adare et al. (PHENIX Collaboration), Phys.Rev.Lett. 107 (2011) 252301.

[10] G. Aad et al. (ATLAS Collaboration), Phys.Rev. C86 (2012) 014907.

[11] B. Schenke, S. Jeon and C. Gale, Phys.Rev. C85 (2012) 024901.

[12] C. Gale, S. Jeon, B. Schenke, P. Tribedy and R. Venugopalan, Phys.Rev.Lett. 110 (2013) 012302.

[13] L. P. Csernai, J. Kapusta and L. D. McLerran, Phys.Rev.Lett. 97 (2006) 152303.

[14] R. A. Lacey, N. Ajitanand, J. Alexander, P. Chung, W. Holzmann et al., Phys.Rev.Lett. 98 (2007) 092301.

[15] S. Plumari, V. Greco and L. P. Csernai, arXiv:1304.6566 [nucl-th].

[16] M. Prakash, M. Prakash, R. Venugopalan and G. Welke, Phys.Rept. 227 (1993) 321-366.

[17] J. W. Chen, Y. H. Li , Y. F. Liu and E. Nakano, Phys.Rev. D76 (2007) 114011.

[18] H. B. Meyer, Phys.Rev. D76 (2007) 101701.

[19] S. K. Das and J. e. Alam, Phys.Rev. D83 (2011) 114011.

[20] S. Plumari, W. M. Alberico, V. Greco and C. Ratti, Phys.Rev. D84 (2011) 094004.

[21] P. B. Arnold , G. D. Moore and L. G. Yaffe, JHEP 0305 (2003) 051.

[22] S. Plumari, A. Puglisi, M. Colonna, F. Scardina and V. Greco, J.Phys. Conf.Ser. 420 (2013) 012029.

[23] S. Plumari, A. Puglisi, F. Scardina and V. Greco, Phys.Rev. C86 (2012) 054902.

[24] M. Ruggieri, F. Scardina, S. Plumari and V. Greco, Phys.Lett. B727 (2013) 177-181.

[25] M. Ruggieri, F. Scardina, S. Plumari and V. Greco, Phys.Rev. C89 (2014) 054914.

[26] S. Plumari, G. L. Guardo, V. Greco and J. Y. Ollitrault, arXiv:1502.04066 [nucl-th].

[27] Z. Xu and C. Greiner, Phys.Rev. C71 (2005) 064901.

[28] B. Zhang, M. Gyulassy and C. M. Ko, Phys.Lett. B455 (1999) 45-48.

[29] D. Molnar and M. Gyulassy, Nucl.Phys. A697 (2002) 495-520.

[30] V. Greco, M. Colonna, M. Di Toro and G. Ferini, Prog.Part.Nucl.Phys. 62 (2009) 562.

[31] S. Plumari, V. Baran, M. Di Toro, G. Ferini and V. Greco, Phys.Lett. B689 (2010) 18-22.

[32] B. Schenke, S. Jeon and C. Gale, Phys.Rev.Lett. 106 (2011) 042301. 